\title{
Hope for the post-antibiotic era?
}

\author{
Randall T. Irvin and Daisy L. Bautista
}

Antibiotic use has substantially improved the quality of life as well as life expectancy itself. Nevertheless, the clinical effectiveness of most antibiotics has declined radically as resistant organisms have become dominant to a point where the use of antibiotics ${ }^{1}$ is seriously compromised. The potential emergence of untreatable "super-bugs"2 has alarmed scientists and clinicians alike, raising the specter of a post-antibiotic era. Such gloomy forecasts have provided added impetus to efforts to develop new strategies for managing infectious diseases. In this issue, Kelly et al. ${ }^{3}$ describe a small clinical trial in which one such antiinfective strategy is successfully employed to block colonization of human teeth by the pathogen Streptococcus mutans.

Some 17 years ago Beachey ${ }^{4}$ first suggested that therapies could be designed to prevent the initial step of infectionnamely, adherence of the pathogen to specific surface receptors (e.g., see Fig. 1). In the present paper, Kelly et al. exploit a mechanism for $S$. mutans pathogenicity by developing a peptide that occludes specific host receptors, thereby preventing pathogen binding and colonization.

Streptococcus mutans is a common oral inhabitant of humans and is the primary cause of dental caries. Its colonization of the tooth surface ${ }^{5}$ begins when a cell surface-localized nonfimbrial adhesin, called streptococcal antigen I/II (SA I/II), binds to salivary agglutinin and other components of the pellicle (a complex layer of glycoproteins bound to the mineral matrix of teeth). Previous studies had demonstrated that inactivating the SA I/II adhesin prevents colonization and caries ${ }^{6}$.

Randall T. Irvin is a member of the Canadian Bacterial Diseases Network and the Protein Engineering Network of Centres of Excellence, and professor in the Department of Medical Microbiology and Immunology, University of Alberta, Edmonton, Alberta, Canada T6G 2H7 (Randy.Irvin@ualberta.ca).DaisyL. Bautista is a graduate student in the same department.
Kelly et al. identify and synthesize a 20mer linear peptide containing a salivary agglutinin receptor-binding domain that effectively inhibits SAI/II binding to salivary agglutinin. This synthetic salivary agglutinin receptor-binding domain is an unstructured peptide that displays a relatively high affinity for its receptor, despite the unfavorable entropic interaction between unstructured peptides and their receptors.

in the oral cavity, and requires extremely high doses to prevent a multivalent ligand (in this case the pathogen) from binding to its receptors. The results of present study, however, provide further evidence for the efficacy of anti-adhesin therapeutic strategies. The critical issue that remains is whether they offer any significant advantage over antibiotics.

Most antibiotics exert strong selective evolutionary pressure on pathogens, thus increasing the probability that resistance will develop. In contrast, anti-adhesin therapeutic strategies could potentially minimize selective evolutionary pressure on the pathogen, as prevention of colonization is not necessarily a selective disadvantage to the organism (assuming that the pathogen has a habitat capability outside of the human host). Thus, the potential for mutational escape or development of "resistance" to this type of therapeutic may be lessened.

Although anti-adhesinbased therapeutic strategies are not yet in clinical practice, this strategy has been shown to be effective for both active and passive vaccines ${ }^{5,7}$ that target and inactivate pathogen adhesins. For the present system at least, the approach of blocking the host receptor is a novel and realistic option. One concern that could limit its applicability, however, is that most pathogens appear to usurp critical host sur-

In a small human trial using a dose of 1 $\mathrm{mg} / \mathrm{ml}$, the authors found that the synthetic salivary agglutinin receptor-binding domain prevented recolonization of human teeth by $S$. mutans for 88 days for one patient and for $>120$ days for the remaining three patients. The peptide appeared to have a short residency time in the oral cavity and thus may exert its protective effect indirectly. A possible mechanism for its action is that it first occludes receptors and prevents $S$. mutans adherence to tooth surfaces. This process would then allow a biofilm to form, ultimately preventing $S$. mutans colonization through microbial competition.

Such observations are intriguing, as one would initially anticipate that a linear unstructured peptide would be a very poor candidate to take into the clinic: the synthetic receptor-binding domain has a very low affinity constant for its receptor, degrades rapidly face components to use as receptors. Consequently, receptor-blocking ligands could conceivably induce or block host signaling pathways, thereby eliciting undesired host responses.

That said, however, the pressing need for alternative infectious disease therapeutic strategies justifies a serious examination of the receptor-blocking strategy. Whether the results of Kelly et al. will eventually eliminate a substantial amount of unpleasant time in a dental chair is a question that will have to await further studies. But we can hope.

1. Neu, H.C. 1992. Science 257:1064-1073.

2. Edmond, M.B., Wenzel, R.P., and Pasculle, A.W. 1996. Ann. Intern. Med. 124:329-334.

3. Kelly, C.G. et al. 1999. Nat. Biotechnol. 17:42-47.

4. Beachey, E.H. 1981. J. Infect. Dis. 143:325-345.

5. Jenkinson, H.F. and Demuth, D.R. 1997. Mol. Microbiol. 23:183-190.

6. Ma, J-K.C. et al. 1989. Clin. Exp. Immunol. 77:331-337.

7. Service, R.F. 1997. Science 276:533. 